\title{
GAUSSIAN EXPANSIONS AND BOUNDS FOR THE POISSON DISTRIBUTION APPLIED TO THE ERLANG B FORMULA
}

\author{
A. J. E. M. JANSSEN, ${ }^{*}$ Philips Research \\ J. S. H. VAN LEEUWAARDEN, ${ }^{* *}$ Eindhoven University of Technology and EURANDOM \\ B. ZWART, ${ }^{* * *}$ Georgia Institute of Technology
}

\begin{abstract}
This paper presents new Gaussian approximations for the cumulative distribution function $\mathrm{P}\left(A_{\lambda} \leq s\right)$ of a Poisson random variable $A_{\lambda}$ with mean $\lambda$. Using an integral transformation, we first bring the Poisson distribution into quasi-Gaussian form, which permits evaluation in terms of the normal distribution function $\Phi$. The quasi-Gaussian form contains an implicitly defined function $y$, which is closely related to the Lambert $W$-function. A detailed analysis of $y$ leads to a powerful asymptotic expansion and sharp bounds on $\mathrm{P}\left(A_{\lambda} \leq s\right)$. The results for $\mathrm{P}\left(A_{\lambda} \leq s\right)$ differ from most classical results related to the central limit theorem in that the leading term $\Phi(\beta)$, with $\beta=(s-\lambda) / \sqrt{\lambda}$, is replaced by $\Phi(\alpha)$, where $\alpha$ is a simple function of $s$ that converges to $\beta$ as $s$ tends to $\infty$. Changing $\beta$ into $\alpha$ turns out to increase precision for small and moderately large values of $s$. The results for $\mathrm{P}\left(A_{\lambda} \leq s\right)$ lead to similar results related to the Erlang B formula. The asymptotic expansion for Erlang's B is shown to give rise to accurate approximations; the obtained bounds seem to be the sharpest in the literature thus far.
\end{abstract}

Keywords: Erlang B formula; Erlang loss model; Poisson distribution; normal distribution; Gaussian integrals; Lambert $W$-function; Ramanujan's conjecture; asymptotic expansion; bounds

2000 Mathematics Subject Classification: Primary 34E05; 60K25; 62E20

\section{Introduction}

Arguably the most famous result in stochastic network theory is the Erlang B formula, derived by A. K. Erlang in 1917. The Erlang B formula gives the steady-state blocking probability in the Erlang loss model (the M/G/s/s queue). This model has $s$ homogeneous servers working in parallel and no extra waiting space. Customers that find all $s$ servers busy upon arrival are blocked (lost). Customers are assumed to arrive according to a Poisson process with rate $\eta$ and require service times that are independent and generally distributed with mean $1 / \mu$. Following convention, we define the offered load as $\lambda=\eta / \mu$, and the server utilization as $\rho=\lambda / s$. The

\footnotetext{
Received 10 October 2007; revision received 29 November 2007.

* Postal address: Philips Research, Digital Signal Processing Group, HTC-36, 5656 AE Eindhoven, The Netherlands. Email address: a.j.e.m.janssen@philips.com

** Postal address: EURANDOM, PO Box 513, 5600 MB Eindhoven, The Netherlands.

Email address: j.s.h.v.leeuwaarden@tue.nl

*** Postal address: Georgia Institute of Technology, H. Milton Stewart School of Industrial and Systems Engineering, 765 Ferst Drive, Atlanta, GA 30332, USA. Email address: bertzwart@ gatech.edu
} 
Erlang B formula is then given by

$$
B(s, \lambda)=\frac{\lambda^{s} / s !}{\sum_{k=0}^{s} \lambda^{k} / k !}=\frac{\mathrm{P}\left(A_{\lambda}=s\right)}{\mathrm{P}\left(A_{\lambda} \leq s\right)},
$$

with $A_{\lambda}$ a Poisson random variable with mean $\lambda$.

With $\beta \in \mathbb{R}$ a constant such that $s=\lambda+\beta \sqrt{\lambda}$, Erlang (see [5]) observed that, for large values of $s$ (and $\lambda$ ), the blocking probability can be very well approximated by

$$
B(s, \lambda) \approx \frac{\phi(\beta)}{\Phi(\beta) \sqrt{\lambda}},
$$

where $\Phi(x)$ and $\phi(x)$ denote the standard normal cumulative distribution function (CDF) and density, respectively. This result follows almost immediately from the central limit theorem for Poisson laws. Since Erlang did not provide a proof, Brockmeyer gave a deduction of this limiting result in [5].

The Erlang B formula has found numerous applications, which increases the importance of a scaling result like (2). In their seminal paper, Halfin and Whitt [10] suggested a similar scaling procedure for queues with many servers and infinite waiting room. The regime where a manyserver queue is both in heavy traffic and critically loaded is known as the Halfin-Whitt regime or the quality and efficiency driven (QED) regime and has been the subject of an extensive recent research effort, motivated by agent staffing problems in customer contact centers; see, for example, [1], [4], [9], [20], and the references therein. The staffing rule $s=\lambda+\beta \sqrt{\lambda}$ is known as square-root staffing.

The present paper is concerned with the derivation of bounds and asymptotic expansions that have the same leading behavior as (2), but that are also sharp for small and moderately large values of $s$. Our results therefore complement the abovementioned works.

When $\lambda$ is a positive integer, $A_{\lambda}$ is the sum of $\lambda$ Poisson random variables with mean 1 . The central limit theorem and the Berry-Esséen bound imply that

$$
\mathrm{P}\left(A_{\lambda} \leq s\right)=\Phi(\beta)+\mathcal{O}\left(\lambda^{-1 / 2}\right) \quad \text { as } \lambda \rightarrow \infty .
$$

To obtain better estimates for the error and to improve on this result, we can derive asymptotic expansions. There are various general theorems that yield asymptotic expansions for $\mathrm{P}\left(A_{\lambda} \leq s\right)$ in ascending positive powers of $\lambda^{-1 / 2}$; see, for example, [2], [3], [7], [11], [16], and [19]. One example would be the Edgeworth expansion, which for the Poisson distribution yields (see [2, Equation (4.18)])

$$
\mathrm{P}\left(A_{\lambda} \leq s\right)=\Phi(\beta)-\frac{\phi(\beta)\left(\beta^{2}-1\right)}{6 \sqrt{\lambda}}+\mathcal{O}\left(\frac{1}{\lambda}\right) .
$$

We shall derive an alternative asymptotic expansion for $\mathrm{P}\left(A_{\lambda} \leq s\right)$ in ascending positive powers of $s^{-1 / 2}$. In contrast to classical expansions related to the central limit theorem, like Edgeworth expansions or saddle point approximations, the leading term in our expansion is not $\Phi(\beta)$. Instead, it is $\Phi(\alpha)$, where $\alpha$ is a function of $s$ (cf. (8), below) that converges to $\beta$ as $s$ tends to $\infty$ (assuming $\beta$ to be fixed).

We shall demonstrate that this switch from $\beta$ to $\alpha$ is very convenient. The first few terms of the expansion serve as sharp approximations to $\mathrm{P}\left(A_{\lambda} \leq s\right)$, even for small and moderate values of $s$. Our expansion is intimately related with the expansion derived in [21] for the incomplete 
gamma function, although the coefficients in the expansion are, except for the leading term, not the same. In his by now classical treatment of the Erlang B formula, Jagerman [12] provided several alternative asymptotic expansions; see Section 5. The main difference between our results and those in [12] and [21] is perhaps the fact that truncated versions of the expansion can be converted into bounds, as explained below. Another difference is that our expansion for Erlang's B is also accurate in the large deviations regime, where the ratio of $s$ and $\lambda$ is fixed.

In passing from the Poisson distribution to its normal approximation, we first bring $\mathrm{P}\left(A_{\lambda} \leq s\right)$ into what we call quasi-Gaussian form (cf. (9), below), which permits evaluation in terms of the normal distribution function $\Phi$. The quasi-Gaussian form contains an implicitly defined function $y$, related to the Lambert $W$-function, which admits a power series representation. This leads to the asymptotic expansion for $\mathrm{P}\left(A_{\lambda} \leq s\right)$.

The idea of bringing $\mathrm{P}\left(A_{\lambda} \leq s\right)$ into quasi-Gaussian form has been introduced by the authors in their recent paper [13] on corrected asymptotics in the Halfin-Whitt regime for the delay probability in the $\mathrm{M} / \mathrm{D} / \mathrm{s}$ queue. In [13] a detailed analysis of $y$ was presented for the case in which $\lambda<s$. The present setting requires additional analysis for the case in which $\lambda \geq s$. Moreover, in the present paper we fully exploit the fact that the quasi-Gaussian form permits us to derive bounds on $\mathrm{P}\left(A_{\lambda} \leq s\right)$ by deriving bounds on $y$ and its derivative $y^{\prime}$. The bounds on $\mathrm{P}\left(A_{\lambda} \leq s\right)$ are of the Berry-Esséen type, except that we again express our approximation in terms of $\alpha$ instead of $\beta$. Using the Berry-Esséen theorem, Michel [18] proved that

$$
\left|\mathrm{P}\left(A_{\lambda} \leq s\right)-\Phi(\beta)\right| \leq \frac{0.8}{\sqrt{\lambda}} .
$$

Our bounds will turn out to be much sharper.

The results for $\mathrm{P}\left(A_{\lambda} \leq s\right)$ lead to corresponding results for Erlang's $\mathrm{B}$. The asymptotic expansion for Erlang's B is shown to give rise to accurate approximations and the bounds seem to be the sharpest obtained in the literature thus far. The following result is among the most appealing ones obtained in this paper.

Theorem 1. For $\lambda>0$ and $s \in \mathbb{N}$, the reciprocal of Erlang's $B$ is bounded by

$$
\begin{aligned}
& B(s, \lambda)^{-1} \leq \frac{\Phi(\alpha) \sqrt{s}}{\phi(\alpha)}+\frac{2}{3}+\frac{\sqrt{s}}{\phi(\alpha)(12 s-1)}, \\
& B(s, \lambda)^{-1} \geq \frac{\Phi(\alpha) \sqrt{s}}{\phi(\alpha)}+\frac{2}{3},
\end{aligned}
$$

with $\alpha$ defined as in (8), below.

Let us compare these bounds to Erlang's approximation (2). Since $\alpha \uparrow \beta$ and $\lambda \uparrow s$ as $s$ tends to $\infty$, we conclude that the bounds in Theorem 1 have the same leading term as in (2). However, changing $\beta$ into $\alpha$ turns out to increase precision for moderately large values of $s$. The accuracy of the bounds is improved further by the first-order correction term $\frac{2}{3}$.

One attractive feature of the bounds is that they are expressed in just one parameter $\alpha$, which is a simple function of $\lambda$ and $s$. Hence, every pair $(\lambda, s)$ is replaced by one parameter $\alpha$, which makes the bounds as simple as (2), but much more effective.

While visual inspection of the bounds in Theorem 1 already suggests good accuracy, we present a framework within which even sharper bounds can be obtained. These bounds, again just in terms of the parameter $\alpha$, involve higher-order correction terms. We shall also indicate how our framework may lead to a proof and sharpening of a conjecture of Ramanujan on the exponential function. 
We structure the paper as follows. In Section 2 we present the quasi-Gaussian form for the $\mathrm{CDF}$ of the Poisson distribution and derive an expansion for $\mathrm{P}\left(A_{\lambda} \leq s\right)$ in terms of Gaussian integrals. In Section 3 we derive bounds on $\mathrm{P}\left(A_{\lambda} \leq s\right)$, valid for all $\lambda$ and $s$. In Section 4 we derive sharper bounds on $\mathrm{P}\left(A_{\lambda} \leq s\right)$, separately for $\lambda \geq s$ and $\lambda<s$. In Section 5 all results for Erlang's B are presented, and in Section 6 we provide a discussion on Ramanujan's conjecture. Some concluding remarks are made in Section 7. In Appendix A we describe various ways to evaluate the crucial function $y$.

\section{Quasi-Gaussian form for the Poisson distribution}

From the relation between the Poisson distribution and the incomplete gamma function, we obtain

$$
\begin{aligned}
\mathrm{P}\left(A_{\lambda} \leq s\right) & =\sum_{j=0}^{s} \mathrm{e}^{-\lambda} \frac{\lambda^{j}}{j !} \\
& =\frac{1}{s !} \int_{\lambda}^{\infty} \mathrm{e}^{-t} t^{s} \mathrm{~d} t \\
& =\frac{p(s) \sqrt{s}}{\sqrt{2 \pi}} \int_{\rho}^{\infty} \mathrm{e}^{s(1-u+\ln u)} \mathrm{d} u,
\end{aligned}
$$

with $\rho=\lambda / s$ and $p(s)=s^{s} \mathrm{e}^{-s} \sqrt{2 \pi s} / s$ !. Then consider the equation

$$
f(y):=-y-\ln (1-y)=\frac{1}{2} x^{2}
$$

with $x \in \mathbb{C}$, from which $y$ is to be solved. We note that

$$
f(y)=\frac{1}{2} y^{2}+\frac{1}{3} y^{3}+\frac{1}{4} y^{4}+\cdots,
$$

whence there is an analytic solution $y(x)$ around $x=0$ that satisfies

$$
y(x)=x+\mathcal{O}\left(x^{2}\right) \quad \text { as } x \rightarrow 0 .
$$

We choose, for $x \in \mathbb{R}$, the function $y(x)$ to be the root of (7) with the same sign as $x$. Clearly, by separate consideration of $x \in(-\infty, 0)$ and $x \in(0, \infty)$, we have that $y$ increases in $x \in \mathbb{R}$, from $-\infty$ at $x=-\infty$ to 1 at $x=\infty$; see Figure 1 . Hence, for any $x \in \mathbb{R}$, there exists a unique solution $y(x)=y$ of (7). Let

$$
\alpha=\sqrt{-2 s(1-\rho+\ln \rho)}, \quad \operatorname{sgn}(\alpha)=\operatorname{sgn}(1-\rho) .
$$

Then, using

$$
s(1-\rho+\ln \rho)=-\frac{1}{2} \alpha^{2},
$$

we arrive from (6) at the following result for the Poisson distribution.

Lemma 1. For $\lambda>0$ and $s \in \mathbb{N}$, the CDF of the Poisson distribution can be represented as

$$
\mathrm{P}\left(A_{\lambda} \leq s\right)=\frac{p(s)}{\sqrt{2 \pi}} \int_{-\infty}^{\alpha} \mathrm{e}^{-x^{2} / 2} y^{\prime}\left(\frac{x}{\sqrt{s}}\right) \mathrm{d} x .
$$




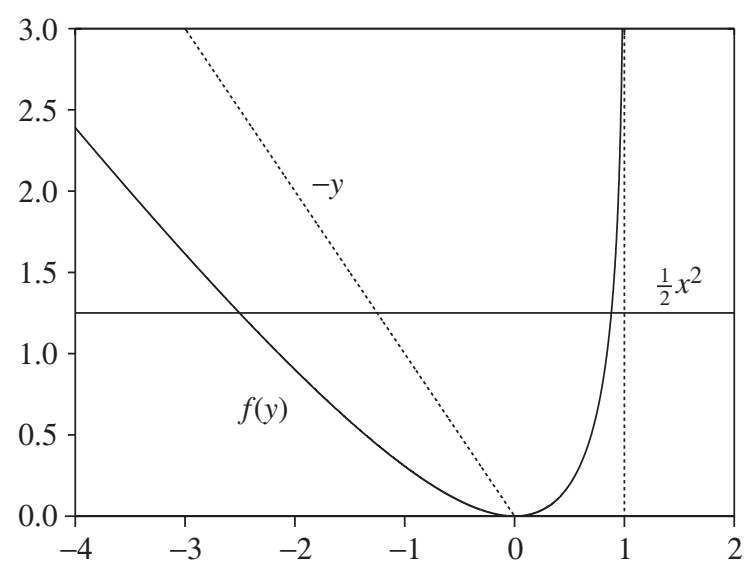

Figure 1: The function $f(y)$.

In [13] we have proved that $y$ admits the power series representation

$$
y(x)=\sum_{n=1}^{\infty} a_{n} x^{n}, \quad|x|<2 \sqrt{\pi},
$$

with $a_{1}=1$ and the $a_{n}$ s recursively defined as

$$
a_{k+2}=\frac{-1}{k+3}\left(a_{k+1}+\sum_{n=1}^{k}(n+1) a_{n+1} a_{k+2-n}\right), \quad k=0,1, \ldots
$$

The first five coefficients of $a_{n}$ are given by

$$
a_{1}=1, \quad a_{2}=-\frac{1}{3}, \quad a_{3}=\frac{1}{36}, \quad a_{4}=\frac{1}{270}, \quad a_{5}=\frac{1}{4320} .
$$

Combining the quasi-Gaussian form and the power series representation gives an asymptotic expansion for the Poisson distribution.

Theorem 2. For $s=\lambda+\beta \sqrt{\lambda}$ with $\beta$ some fixed real number, there exists, as $s$ tends to $\infty, a$ representation of the form

$$
\mathrm{P}\left(A_{\lambda} \leq s\right) \sim p(s) \sum_{n=0}^{\infty}(n+1) a_{n+1} \chi_{n}(\alpha) s^{-n / 2},
$$

where

$$
\chi_{n}(\alpha)=\frac{1}{\sqrt{2 \pi}} \int_{-\infty}^{\alpha} x^{n} \mathrm{e}^{-x^{2} / 2} \mathrm{~d} x
$$

with $\alpha$ defined as in (8) and $a_{n}$ defined as in (11).

The ' $\sim$ ' in (13) is the commonly used symbol for asymptotic equivalence: for any $N=$ $0,1, \ldots$, we have

$$
\mathrm{P}\left(A_{\lambda} \leq s\right)-p(s) \sum_{n=0}^{N}(n+1) a_{n+1} \chi_{n}(\alpha) s^{-n / 2}=\mathcal{O}\left(s^{-(N+1) / 2}\right), \quad s \rightarrow \infty .
$$


In the present case it can be shown, by elementary (but lengthy) computations, that $\mathcal{O}$ holds for $s \geq|\alpha| / \pi a^{2}$ and that the constant implied by the $\mathcal{O}$ can be bounded by

$$
\frac{5}{a(1-a / 2)}\left(\frac{N+1}{\pi \mathrm{e} a^{2}}\right)^{(N+1) / 2} .
$$

Here $a$ is any number in the interval $(0,2)$.

Note that, with our conventions,

$$
s=\lambda+\beta \sqrt{\lambda} \Longleftrightarrow \lambda=s+\frac{1}{2} \beta^{2}-\beta \sqrt{s+\frac{\beta^{2}}{4}} .
$$

The expansion in (13) starts as

$$
\mathrm{P}\left(A_{\lambda} \leq s\right) \sim p(s)\left(\Phi(\alpha)+\frac{2}{3 \sqrt{s}} \phi(\alpha)+\frac{1}{12 s}(\Phi(\alpha)-\alpha \phi(\alpha))\right),
$$

and is fully described in terms of $\alpha$ and Gaussian integrals. In fact, the first six values of $\chi_{n}$ (suppressing the $\alpha$ ) are

$$
\begin{array}{ll}
\chi_{0}=\Phi(\alpha), & \chi_{1}=-\phi(\alpha), \\
\chi_{2}=\Phi(\alpha)-\alpha \phi(\alpha), & \chi_{3}=-\left(2+\alpha^{2}\right) \phi(\alpha), \\
\chi_{4}=3 \Phi(\alpha)-\alpha\left(3+\alpha^{2}\right) \phi(\alpha), & \chi_{5}=-\left(8+4 \alpha^{2}+\alpha^{4}\right) \phi(\alpha) .
\end{array}
$$

\section{General bounds}

From the quasi-Gaussian form (9) we can conclude that bounds on $y^{\prime}$ lead to bounds on $\mathrm{P}\left(A_{\lambda} \leq s\right)$. Figure 2 depicts $y^{\prime}$ for $x \in[-2,3]$. In this section we shall derive bounds on $y^{\prime}$ that hold for all $x \in \mathbb{R}$ and will lead to bounds on $\mathrm{P}\left(A_{\lambda} \leq s\right)$ that hold for every pair $(\lambda, s)$. As shown by Theorem 4 , the accuracy of some of these bounds is closely related to shifting the mean in estimating the Poisson distribution by a Gaussian distribution.

We first provide two lemmas that are useful in proving bounds on $y$ and $y^{\prime}$.

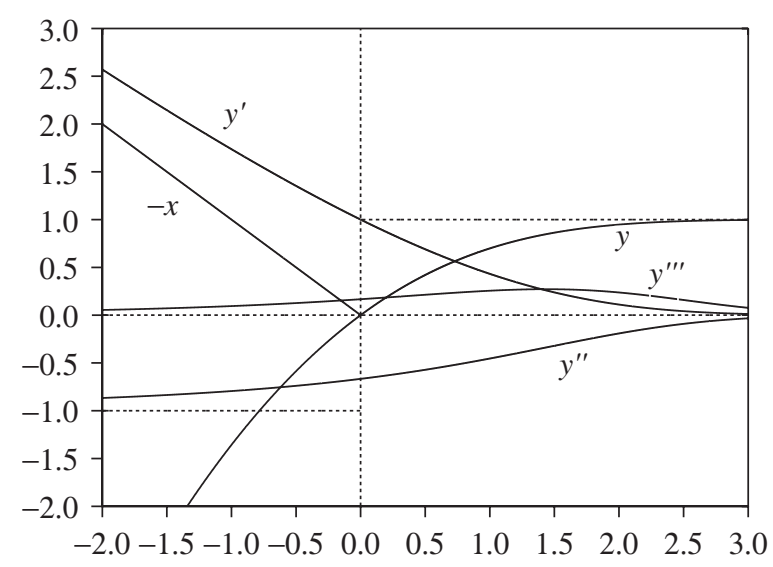

FIgure 2: The function $y(x)$ and its derivatives. 
Lemma 2. Let I be an interval of the form $\left(-x_{1}, x_{2}\right),\left(-x_{1}, 0\right]$, or $\left[0, x_{2}\right)$, where $x_{1}, x_{2}>0$. Assume that $F$ is smooth on $I$ and that $F(0)=0$ and $F(x)<1, x \in I$. Then

$$
\begin{array}{ll}
\frac{F^{\prime}(x) F(x)}{1-F(x)} \geq x, & x \in I \quad \Longrightarrow \quad y(x) \leq F(x), \quad x \in I, \\
\frac{F^{\prime}(x) F(x)}{1-F(x)} \leq x, & x \in I \Longrightarrow y(x) \geq F(x), \quad x \in I .
\end{array}
$$

Proof. Consider the case in which $I=\left[0, x_{2}\right)$ with $x_{2}>0$. Then, for $u \in I$, we have (see Figure 1)

$$
y(u) \leq F(u) \Longleftrightarrow \frac{1}{2} u^{2} \leq-F(u)-\ln (1-F(u)) .
$$

Since $y(0)=F(0)=0$, the right member of (16) holds true for $u \in I$ when

$$
\begin{aligned}
x=\frac{\mathrm{d}}{\mathrm{d} x}\left(\frac{1}{2} x^{2}\right) & \leq \frac{\mathrm{d}}{\mathrm{d} x}(-F(x)-\ln (1-F(x))) \\
& =\frac{F^{\prime}(x) F(x)}{1-F(x)}, \quad 0 \leq x \leq u .
\end{aligned}
$$

From this, (14) follows and (15) follows similarly.

Next consider the case in which $I=\left(-x_{1}, 0\right]$ with $x_{1}>0$. Then, for $v \in I$, we have (see Figure 1)

$$
y(v) \leq F(v) \Longleftrightarrow \frac{1}{2} v^{2} \geq-F(v)-\ln (1-F(v)) .
$$

Since $y(0)=F(0)=0$, the right member of (17) holds true for $v \in I$ when

$$
\begin{aligned}
x=\frac{\mathrm{d}}{\mathrm{d} x}\left(\frac{1}{2} x^{2}\right) & \leq \frac{\mathrm{d}}{\mathrm{d} x}(-F(x)-\ln (1-F(x))) \\
& =\frac{F^{\prime}(x) F(x)}{1-F(x)}, \quad v \leq x \leq 0 .
\end{aligned}
$$

From this, (14) follows and (15) follows similarly.

Lemma 3. Let I be an interval of the form $\left(-x_{1}, x_{2}\right),\left(-x_{1}, 0\right]$, or $\left[0, x_{2}\right)$, where $x_{1}, x_{2}>0$. Assume that $f$ is smooth on I and that $f(0)=1, f(x)>0$, and $x+f(x)>0, x \in I$. Then

$$
\begin{aligned}
& \frac{1-x f^{\prime}(x) / f(x)}{(x+f(x))^{2}} \leq 1, \quad x \in I \quad \Longrightarrow \quad y^{\prime}(x) \leq f(x), \quad x \in I, \\
& \frac{1-x f^{\prime}(x) / f(x)}{(x+f(x))^{2}} \geq 1, \quad x \in I \quad \Longrightarrow \quad y^{\prime}(x) \geq f(x), \quad x \in I .
\end{aligned}
$$

Proof. Consider the case in which $I=\left[0, x_{2}\right)$ with $x_{2}>0$. From (7) we obtain, by differentiation with respect to $x$ and some rewriting of the equation,

$$
y^{\prime}(x)=\frac{x}{y(x)}-x
$$

For $u \in I$, the inequality $y^{\prime}(u) \leq f(u)$ then becomes

$$
y(u) \geq \frac{u}{u+f(u)}=: s(u) .
$$


By our assumptions we have $s(u) \in(0,1)$; hence, by monotonicity of $t \in(0,1) \mapsto-t-$ $\ln (1-t)$, inequality (20) is equivalent to

$$
-s(u)-\ln (1-s(u)) \leq \frac{1}{2} u^{2}=-y(u)-\ln (1-y(u)) .
$$

Since $s(0)=y(0)=0$, inequality (21) holds true for $u \in I$ when

$$
\frac{\mathrm{d}}{\mathrm{d} x}(-s(x)-\ln (1-s(x)))=\frac{s^{\prime}(x) s(x)}{1-s(x)} \leq x, \quad 0 \leq x \leq u .
$$

From this, (18) follows and (19) follows similarly. The same results for the domain $I=\left(-x_{1}, 0\right]$ with $x_{1}>0$ follow along similar lines as the case dealt with above.

Warning: when the condition $F(x)<1$ in Lemma 2 or the conditions $f(x)+x>0$ and $f(x)>0$ in Lemma 3 hold in a disconnected set, the corresponding inequality for $y$ or $y^{\prime}$ may fail to hold in the components not containing 0 .

With the aid of Lemma 2 we can easily show that, for example,

$$
x-\frac{1}{2} x^{2} \leq y(x) \leq x, \quad x \in \mathbb{R} ; \quad y(x) \leq \min \left\{x-\frac{1}{3} x^{2},-\frac{1}{2} x^{2}\right\}, \quad x \leq 0 .
$$

For $y^{\prime}$, we find the following useful bounds.

Lemma 4. We have

$$
1-\frac{2}{3} x \leq y^{\prime}(x) \leq \mathrm{e}^{-2 x / 3}, \quad x \in \mathbb{R} .
$$

Proof. In Lemma 3 choose $f(x)=\mathrm{e}^{-2 x / 3}$. This $f$ satisfies $f(x)>0, x+f(x)>0$, and $f(0)=1$. We compute

$$
\frac{1-x f^{\prime}(x) / f(x)}{(x+f(x))^{2}}=\frac{1+2 x / 3}{\left(x+\mathrm{e}^{-2 x / 3}\right)^{2}}, \quad x \in \mathbb{R} .
$$

We want to verify that (24) is less than or equal to 1 . To that end we can assume that $1+\frac{2}{3} x \geq 0$. Now,

and it follows that

$$
1+\frac{2}{3} x \leq\left(1+\frac{1}{3} x\right)^{2}, \quad x+\mathrm{e}^{-2 x / 3} \geq 1+\frac{1}{3} x,
$$

$$
\frac{1+2 x / 3}{\left(x+\mathrm{e}^{-2 x / 3}\right)^{2}} \leq\left(\frac{1+x / 3}{1+x / 3}\right)^{2}=1,
$$

as required.

The inequality $1-\frac{2}{3} x \leq y^{\prime}(x)$ was proved in [13] for $x \geq 0$. To prove it for $x \in \mathbb{R}$, we choose $f(x)=1-\frac{2}{3} x$ in Lemma 3. We compute

$$
\frac{1-x f^{\prime}(x) / f(x)}{(x+f(x))^{2}}=\frac{1}{1-2 x / 3} \frac{1}{(1+x / 3)^{2}},
$$

which is to be considered for those $x$ for which $f(x)>0$ and $x+f(x)>0$, i.e. for $x \in\left(-3, \frac{3}{2}\right)$. This is sufficient since $y^{\prime}(x)>0 \geq 1-\frac{2}{3} x, x \geq \frac{3}{2}$, and $y^{\prime}(x) \geq-x>1-\frac{2}{3} x, x \leq-3$. Note that

$$
\left(1-\frac{2}{3} x\right)\left(1+\frac{1}{3} x\right)^{2}=1-\frac{1}{3} x^{2}-\frac{2}{27} x^{3}
$$

has derivative $-\frac{2}{3} x\left(1+\frac{1}{3} x\right)$. Therefore, the maximum of (25) on $x \in\left(-3, \frac{3}{2}\right)$ equals 1 (assumed at $x=0)$. Thus,

$$
\frac{1-x f^{\prime}(x) / f(x)}{(x+f(x))^{2}} \geq 1, \quad x \in\left(-3, \frac{3}{2}\right) .
$$

By Lemma 3, this completes the proof. 
Theorem 3. For $\lambda>0$ and $s \in \mathbb{N}$, the CDF of the Poisson distribution is bounded by

$$
\begin{aligned}
& \mathrm{P}\left(A_{\lambda} \leq s\right) \leq 1-p(s)\left(\Phi(-\alpha)-\frac{2}{3 \sqrt{s}} \phi(\alpha)\right), \\
& \mathrm{P}\left(A_{\lambda} \leq s\right) \geq p(s)\left(\Phi(\alpha)+\frac{2}{3 \sqrt{s}} \phi(\alpha)\right) .
\end{aligned}
$$

Proof. Substituting the lower bound in (23) into the quasi-Gaussian form (9) leads to

$$
\mathrm{P}\left(A_{\lambda} \leq s\right) \geq \frac{p(s)}{\sqrt{2 \pi}} \int_{-\infty}^{\alpha} \mathrm{e}^{-x^{2} / 2}\left(1-\frac{2}{3 \sqrt{s}} x\right) \mathrm{d} x,
$$

which equals (27). The upper bound (26) follows from the identity

$$
\mathrm{P}\left(A_{\lambda} \leq s\right)=1-\frac{p(s)}{\sqrt{2 \pi}} \int_{\alpha}^{\infty} \mathrm{e}^{-x^{2} / 2} y^{\prime}\left(\frac{x}{\sqrt{s}}\right) \mathrm{d} x,
$$

which, again using $1-\frac{2}{3} x \leq y^{\prime}(x)$, leads to (26).

Theorem 4. For $\lambda>0$ and $s \in \mathbb{N}$, the CDF of the Poisson distribution is bounded by

$$
\begin{aligned}
& \mathrm{P}\left(A_{\lambda} \leq s\right) \leq p(s) \mathrm{e}^{2 / 9 s} \Phi\left(\alpha+\frac{2}{3 \sqrt{s}}\right) \\
& \mathrm{P}\left(A_{\lambda} \leq s\right) \geq 1-p(s) \mathrm{e}^{2 / 9 s}\left(1-\Phi\left(\alpha+\frac{2}{3 \sqrt{s}}\right)\right) .
\end{aligned}
$$

Proof. The upper bound $y^{\prime}(x) \leq \mathrm{e}^{-2 x / 3}$ in (23) together with (9) leads to

$$
\mathrm{P}\left(A_{\lambda} \leq s\right) \leq \frac{p(s)}{\sqrt{2 \pi}} \int_{-\infty}^{\alpha} \exp \left(-\frac{1}{2} x^{2}-\frac{2}{3 \sqrt{s}} x\right) \mathrm{d} x,
$$

which equals (29). The upper bound $y^{\prime}(x) \leq \mathrm{e}^{-2 x / 3}$ and (28) give (30).

Table 1 displays some results for the above bounds for $s=10$. While all bounds are sharp for the case in which $\lambda \leq s$, they tend to be less accurate for the case in which $\lambda>s$. This is resolved in Section 4.

Let us close this section with the following Berry-Esséen-type result (compare with (3)).

Corollary 1. For $\lambda>0$ and $s \in \mathbb{N}$, we have

$$
0 \leq \mathrm{e}^{2 / 9 s} \Phi\left(\alpha+\frac{2}{3 \sqrt{s}}\right)-\mathrm{P}\left(A_{\lambda} \leq s\right) \leq \mathrm{e}^{2 / 9 s}-1,
$$

where $\mathrm{e}^{2 / 9 s}-1=\frac{2}{9} s^{-1}+\mathcal{O}\left(s^{-2}\right)$.

Proof. The bounds (29) and (30) immediately yield

$$
0 \leq p(s) \mathrm{e}^{2 / 9 s} \Phi\left(\alpha+\frac{2}{3 \sqrt{s}}\right)-\mathrm{P}\left(A_{\lambda} \leq s\right) \leq p(s) \mathrm{e}^{2 / 9 s}-1,
$$

where $p(s) \mathrm{e}^{2 / 9 s}-1=\frac{5}{36} s^{-1}+\mathcal{O}\left(s^{-2}\right)$. Using $p(s) \leq 1$, results in (31). 
TABLE 1: Bounds on $\mathrm{P}\left(A_{\lambda} \leq s\right)$ for $s=10$.

\begin{tabular}{rrccccc}
\hline$\lambda$ & $\alpha$ & $\mathrm{P}\left(A_{\lambda} \leq s\right)$ & $(26)$ & $(27)$ & $(29)$ & $(30)$ \\
\hline 1 & 5.2964 & 1.0000 & 1.0000 & 0.9917 & 1.0140 & 1.0000 \\
2 & 4.0235 & 1.0000 & 1.0000 & 0.9917 & 1.0140 & 1.0000 \\
3 & 3.1748 & 0.9997 & 0.9998 & 0.9915 & 1.0136 & 0.9996 \\
4 & 2.5151 & 0.9972 & 0.9976 & 0.9893 & 1.0107 & 0.9967 \\
5 & 1.9654 & 0.9863 & 0.9876 & 0.9793 & 0.9990 & 0.9850 \\
6 & 1.4888 & 0.9574 & 0.9598 & 0.9515 & 0.9688 & 0.9548 \\
7 & 1.0647 & 0.9015 & 0.9050 & 0.8967 & 0.9115 & 0.8975 \\
8 & 0.6803 & 0.8159 & 0.8201 & 0.8118 & 0.8250 & 0.8110 \\
9 & 0.3274 & 0.7060 & 0.7105 & 0.7022 & 0.7147 & 0.7007 \\
10 & 0.0000 & 0.5830 & 0.5876 & 0.5793 & 0.5916 & 0.5777 \\
11 & -0.3063 & 0.4599 & 0.4644 & 0.4561 & 0.4684 & 0.4545 \\
12 & -0.5946 & 0.3472 & 0.3519 & 0.3437 & 0.3555 & 0.3415 \\
13 & -0.8676 & 0.2517 & 0.2568 & 0.2485 & 0.2592 & 0.2453 \\
14 & -1.1272 & 0.1757 & 0.1812 & 0.1729 & 0.1823 & 0.1683 \\
15 & -1.3750 & 0.1185 & 0.1246 & 0.1163 & 0.1239 & 0.1099 \\
16 & -1.6124 & 0.0774 & 0.0840 & 0.0757 & 0.0816 & 0.0677 \\
17 & -1.8405 & 0.0491 & 0.0562 & 0.0479 & 0.0523 & 0.0383 \\
18 & -2.0602 & 0.0304 & 0.0378 & 0.0295 & 0.0327 & 0.0187 \\
19 & -2.2722 & 0.0183 & 0.0260 & 0.0178 & 0.0199 & 0.0059 \\
20 & -2.4773 & 0.0108 & 0.0187 & 0.0104 & 0.0119 & -0.0021 \\
\hline
\end{tabular}

\section{More specific bounds}

In this section we shall derive sharper bounds on $\mathrm{P}\left(A_{\lambda} \leq s\right)$. In order to do so, we derive bounds on $y^{\prime}$ that hold only for $x \leq 0$ and that will lead to bounds on $\mathrm{P}\left(A_{\lambda} \leq s\right)$, separately for $\lambda \geq s$ and $\lambda<s$.

Lemma 5. The function y is increasing and concave, and its derivative $y^{\prime}$ is positive, decreasing, and convex.

Proof. We first prove that $y^{\prime \prime}<0$. From (10) and (12), we see that $y^{\prime \prime}(0)=-\frac{2}{3}<0$. So, assume that $x \neq 0$. From $y^{\prime}=x / y-x$ we have

$$
y^{\prime \prime}=\left(\frac{1}{y}-1\right)\left(1-\frac{x^{2}}{y^{2}}\right) .
$$

Clearly, $1 / y-1>0$ when $x \in(0, \infty)$ (i.e. $y \in(0,1))$ and $1 / y-1<0$ when $x \in(-\infty, 0)$ (i.e. $y \in(-\infty, 0))$. Therefore, $y^{\prime \prime}<0$ is equivalent to $y(x)<x$ and this is one of the bounds noted in (22) for $y$.

We shall now prove that $y^{\prime \prime \prime}>0$. Again, by (10) and (12), we see that $y^{\prime \prime \prime}(0)=\frac{1}{6}>0$, so we assume that $x \neq 0$. From (32) and $y^{\prime}=x / y-x$, we compute

$$
y^{\prime \prime \prime}=-\frac{3 x}{y^{4}}\left(\frac{1}{y}-1\right)\left(y^{2}-x^{2}+\frac{2}{3} x^{2} y\right) .
$$

Noting that the factor $-\left(3 x / y^{4}\right)(1 / y-1)$ is negative, it remains to show that

$$
y^{2}-x^{2}+\frac{2}{3} x^{2} y=\left(y+\frac{1}{3} x^{2}\right)^{2}-\left(x^{2}+\frac{1}{9} x^{4}\right)<0 .
$$


Again we distinguish between $x>0$ and $x<0$. For $x>0$, we have $y>0$, and we should show that

$$
y<-\frac{1}{3} x^{2}+\left(x^{2}+\frac{1}{9} x^{4}\right)^{1 / 2}=: r(x) .
$$

We have (see Figure 1)

$$
y(x)<r(x) \Longleftrightarrow f(r(x))>f(y(x))=\frac{1}{2} x^{2} .
$$

With $v=x^{2}>0$, the latter inequality can be formulated as

$$
-\left(v+\frac{1}{9} v^{2}\right)^{1 / 2}+\frac{1}{3} v-\ln \left(1-\left(v+\frac{1}{9} v^{2}\right)^{1 / 2}+\frac{1}{3} v\right)>\frac{1}{2} v .
$$

We only need to consider (33) for $0<v<3$, since $r(x) \geq 1$ when $x^{2}=v \geq 3$. There is equality at $v=0$, so it is enough to check that the derivative of the left-hand side of (33) is greater than or equal to $\frac{1}{2}$, which, after some manipulation, can be shown to be equivalent to

$$
\frac{1}{9} v^{2}-\frac{1}{3} v\left(v+\frac{1}{9} v^{2}\right)^{1 / 2}<0 .
$$

This is indeed true. For the case in which $x<0$, we must check (since $y<0$ ) that

$$
y>-\frac{1}{3} x^{2}-\left(x^{2}+\frac{1}{9} x^{4}\right)^{1 / 2} .
$$

A sufficient condition for this to hold can be found in a similar fashion as above and reads

$$
\frac{1}{9} v^{2}+\frac{1}{3} v\left(v+\frac{1}{9} v^{2}\right)^{1 / 2}>0
$$

with $v=\frac{1}{2} x^{2}>0$. The latter inequality indeed holds which completes the proof.

Lemma 6. Let $\alpha \leq 0$. Then

$$
y^{\prime}(\alpha)+(x-\alpha) y^{\prime \prime}(\alpha) \leq y^{\prime}(x) \leq y^{\prime}(\alpha)-(x-\alpha), \quad x \leq \alpha .
$$

Proof. Since $y^{\prime}$ is convex, the first inequality holds for all $x \in \mathbb{R}$. As to the second inequality in (34), we first take $\alpha<0$. Now

$$
y^{\prime}(x)+x=x\left(\frac{1}{y(x)}-1\right)+x=\frac{x}{y(x)} \leq \frac{\alpha}{y(\alpha)}=y^{\prime}(\alpha)+\alpha, \quad x \leq \alpha .
$$

The inequality in (35) follows from

$$
y(\alpha)=y\left(\frac{\alpha}{x} x+\left(1-\frac{\alpha}{x}\right) \cdot 0\right) \geq \frac{\alpha}{x} y(x)+\left(1-\frac{\alpha}{x}\right) y(0)=\frac{\alpha}{x} y(x),
$$

where we used the concavity of $y$ and $y(0)=0$. Note that $\alpha<0$ so that

$$
y(\alpha) \geq \frac{\alpha}{x} y(x) \Longleftrightarrow \frac{x}{y(x)} \leq \frac{\alpha}{y(\alpha)} .
$$

The case in which $\alpha<0$ is settled now. The case in which $\alpha=0$ follows from the continuity of all functions involved in (34) and letting $\alpha \uparrow 0$.

Substituting the bounds in (34) into the quasi-Gaussian form (9) leads to the following theorem. 
Theorem 5. For $\lambda \geq s \in \mathbb{N}$, and hence $\alpha \leq 0$, the CDF of the Poisson distribution is bounded by

$$
\begin{aligned}
& \mathrm{P}\left(A_{\lambda} \leq s\right) \leq p(s) y^{\prime}(\alpha) \Phi(\alpha)+p(s)(\alpha \Phi(\alpha)+\phi(\alpha)), \\
& \mathrm{P}\left(A_{\lambda} \leq s\right) \geq p(s) y^{\prime}(\alpha) \Phi(\alpha)-p(s) y^{\prime \prime}(\alpha)(\alpha \Phi(\alpha)+\phi(\alpha)) .
\end{aligned}
$$

The difference between the two bounds is given by

$$
p(s)\left(1+y^{\prime \prime}(\alpha)\right)(\alpha \Phi(\alpha)+\phi(\alpha)) .
$$

Using the tail estimate for the Gaussian distribution,

$$
\frac{x}{x^{2}+1} \phi(x) \leq \Phi(-x) \leq \frac{1}{x} \phi(x), \quad x \geq 0,
$$

this difference can be bounded by

$$
0 \leq p(s)\left(1+y^{\prime \prime}(\alpha)\right)(\alpha \Phi(\alpha)+\phi(\alpha)) \leq \frac{p(s)\left(1+y^{\prime \prime}(\alpha)\right)}{\sqrt{2 \pi}\left(1+\alpha^{2}\right)} \mathrm{e}^{-\alpha^{2} / 2} .
$$

The factor $1+y^{\prime \prime}(\alpha)$ on the right-hand side of (36) can furthermore be bounded as follows. We have $0 \leq 1+y^{\prime \prime}(\alpha) \leq \frac{1}{3}, \alpha \leq 0$, and $1+y^{\prime \prime}(\alpha)=\mathcal{O}\left(1 / \alpha^{2}\right)$ as $\alpha \rightarrow-\infty$. Indeed, $y^{\prime \prime \prime}>0$, so $1+y^{\prime \prime}(-\infty) \leq 1+y^{\prime \prime}(\alpha) \leq 1+y^{\prime \prime}(0)=\frac{1}{3}$, and from (32) and the inequalities in (22) for $y$, we obtain $y^{\prime \prime}(-\infty)=-1$ and $1+y^{\prime \prime}(\alpha)=\mathcal{O}\left(1 / \alpha^{2}\right)$.

From (10) it follows that $y^{\prime}$ has the power series representation

$$
y^{\prime}(x)=1-\frac{2}{3} x+\frac{1}{12} x^{2}+\frac{2}{135} x^{3}+\frac{1}{864} x^{4}+\cdots, \quad|x|<2 \sqrt{\pi},
$$

from which we see that (34) for $\alpha=0$ leads to

$$
1-\frac{2}{3} x \leq y^{\prime}(x) \leq 1-x, \quad x \leq 0 .
$$

We now use the coefficients of the power series in (37) to guess and prove bounds on $y^{\prime}$ in terms of polynomials of larger degrees.

Lemma 7. We have

$$
y^{\prime}(x) \leq 1-\frac{2}{3} x+\frac{1}{12} x^{2}, \quad x \leq 0 .
$$

Proof. We apply Lemma 3 with $f(x)=1-\frac{2}{3} x+\frac{1}{12} x^{2}$. We have $f(x)+x>0$ and $f(x) \geq 0$ for $x \leq 0$. The inequality to be shown becomes

$$
\left(1+\frac{1}{3} x+\frac{1}{12} x^{2}\right)^{2}\left(1-\frac{2}{3} x+\frac{1}{12} x^{2}\right) \geq 1-\frac{1}{12} x^{2}, \quad x \leq 0 .
$$

An elementary computation shows that the left-hand side of (39) equals

$$
1-\frac{1}{12} x^{2}-x^{3}\left(\frac{2}{27}+\frac{1}{144} x-\frac{1}{1728} x^{3}\right), \quad x \leq 0,
$$

where the expression in the brackets has its minimum value, $\frac{7}{216}$, at $x=-2$. This completes the proof.

Lemma 8. We have

$$
y^{\prime}(x) \geq 1-\frac{2}{3} x+\frac{1}{12} x^{2}+\frac{2}{135} x^{3}, \quad x \leq 0
$$


Proof. Let

$$
w(x)=x-\frac{1}{3} x^{2}+\frac{1}{36} x^{3}+\frac{1}{270} x^{4} .
$$

For the bound in (41) to hold, it is sufficient to show that

(i) $y^{\prime}(x) \geq w^{\prime}(x)$ for $-2 \leq x \leq 0$,

(ii) $y^{\prime \prime}(-2) \leq w^{\prime \prime}(-2)$,

(iii) $y^{\prime \prime \prime}(x) \geq w^{\prime \prime \prime}(x)$ for $x \leq-2$.

Evidently, part (iii) follows from $w^{\prime \prime \prime}(x)=\frac{1}{6}+\frac{4}{45} x \leq-\frac{1}{90} \leq 0 \leq y^{\prime \prime \prime}(x)$ for $x \leq-2$. In proving part (i) we apply Lemma 3 with $f(x)=w^{\prime}(x)$. Note that $f(x) \geq f(x)+x, x \leq 0$, and $(f(x)+x)^{\prime}=\frac{1}{3}+\frac{1}{6} x+\frac{2}{45} x^{2} \geq 0$, so $f(x)+x \geq f(-2)-2=\frac{74}{135}>0,-2 \leq x \leq 0$. The inequality to be shown becomes, for $-2 \leq x \leq 0$,

$$
\left(1+\frac{1}{3} x+\frac{1}{12} x^{2}+\frac{2}{135} x^{3}\right)^{2}\left(1-\frac{2}{3} x+\frac{1}{12} x^{2}+\frac{2}{135} x^{3}\right) \leq 1-\frac{1}{12} x^{2}-\frac{4}{135} x^{3} .
$$

The left-hand side of (42) can be written as

$$
1-\frac{1}{12} x^{2}-\frac{4}{135} x^{3}-\frac{1}{144} x^{4}+\left(\frac{1}{12}\right)^{3} x^{6}+\varepsilon\left(\frac{1}{6} x^{2}+\frac{1}{48} x^{4}\right)+\varepsilon^{2}\left(3+\frac{1}{4} x^{2}\right)+\varepsilon^{3},
$$

with $\varepsilon=\frac{2}{135} x^{3}$, and so it remains to be shown that, for $-2 \leq x \leq 0$,

$$
-\frac{1}{144}+\left(\frac{1}{12}\right)^{3} x^{2}+\frac{2}{135}\left(\frac{1}{6} x+\frac{1}{48} x^{3}\right)+\left(\frac{2}{135}\right)^{2}\left(3 x^{2}+\frac{1}{4} x^{4}\right)+\left(\frac{2}{135}\right)^{3} x^{5} \leq 0 .
$$

That (43) holds for $-2 \leq x \leq 0$ follows from the fact that the positive terms of (43) at $x=-2$ add up to $\frac{1}{3}\left(\frac{1}{12}\right)^{2}+\left(\frac{\overline{8}}{135}\right)^{2}$, which is smaller than $\frac{1}{144}$. Finally, in proving part (ii) we first observe that $w^{\prime \prime}(-2)=-\frac{37}{45} \approx-0.8222$ and that (see (32))

$$
y^{\prime \prime}(-2)=\left(\frac{1}{y(-2)}-1\right)\left(1-\frac{4}{y^{2}(-2)}\right) \text {. }
$$

Next, from $-y-\ln (1-y)=\frac{1}{2} x^{2}$ and $3.5-\ln 4.5<2<4-\ln 5$, it follows that $y(-2) \in$ $[-3.5,-4.0]$. The cubic $(t-1)\left(1-4 t^{2}\right)$ has a negative derivative when $t \in[-1 / 3.5,-1 / 4.0]$. Therefore,

$$
y^{\prime \prime}(-2) \leq\left(\frac{1}{-3.5}-1\right)\left(1-\frac{4}{(-3.5)^{2}}\right)=-\frac{297}{343} \approx-0.8659 .
$$

This completes the proof.

We arrive at another theorem on the Poisson distribution by substituting (38) and (41) into the quasi-Gaussian form (9).

Theorem 6. For $\lambda \geq s \in \mathbb{N}$, the CDF of the Poisson distribution is bounded by

$$
\begin{aligned}
& \mathrm{P}\left(A_{\lambda} \leq s\right) \leq p(s)\left(\Phi(\alpha)+\frac{2}{3 \sqrt{s}} \phi(\alpha)+\frac{1}{12 s}(\Phi(\alpha)-\alpha \phi(\alpha))\right) \\
& \mathrm{P}\left(A_{\lambda} \leq s\right) \geq p(s)\left(\Phi(\alpha)+\frac{2}{3 \sqrt{s}} \phi(\alpha)+\frac{1}{12 s}(\Phi(\alpha)-\alpha \phi(\alpha))-\frac{2}{135 s^{3 / 2}}\left(2+\alpha^{2}\right) \phi(\alpha)\right) .
\end{aligned}
$$


For the case in which $n=\lambda=s$, we have $\alpha=0$ and

$$
\mathrm{P}\left(A_{n} \leq n\right)=\frac{p(n)}{\sqrt{2 \pi}} \int_{-\infty}^{0} \mathrm{e}^{-x^{2} / 2} y^{\prime}\left(\frac{x}{\sqrt{n}}\right) \mathrm{d} x .
$$

From Theorem 6 we obtain

$$
\begin{aligned}
& \mathrm{P}\left(A_{n} \leq n\right) \leq p(n)\left(\frac{1}{2}+\frac{2}{3 \sqrt{2 \pi n}}+\frac{1}{24 n}\right) \\
& \mathrm{P}\left(A_{n} \leq n\right) \geq p(n)\left(\frac{1}{2}+\frac{2}{3 \sqrt{2 \pi n}}+\frac{1}{24 n}-\frac{4}{135 \sqrt{2 \pi} n^{3 / 2}}\right) .
\end{aligned}
$$

For the case in which $\lambda<s$, we have $\alpha>0$ and

$$
\begin{aligned}
\mathrm{P}\left(A_{\lambda} \leq s\right)= & \frac{p(s)}{\sqrt{2 \pi}} \int_{-\infty}^{0} \mathrm{e}^{-x^{2} / 2} y^{\prime}\left(\frac{x}{\sqrt{s}}\right) \mathrm{d} x \\
& +\frac{p(s)}{\sqrt{2 \pi}} \int_{0}^{\alpha} \mathrm{e}^{-x^{2} / 2} y^{\prime}\left(\frac{x}{\sqrt{s}}\right) \mathrm{d} x .
\end{aligned}
$$

The second integral in (46) can thus be bounded using (23) and

$$
\begin{gathered}
\frac{1}{\sqrt{2 \pi}} \int_{0}^{\alpha} \mathrm{e}^{-x^{2} / 2}\left(1-\frac{2}{3 \sqrt{s}} x\right) \mathrm{d} x=\Phi(\alpha)-\frac{1}{2}-\frac{2}{3 \sqrt{s}}\left(\frac{1}{\sqrt{2 \pi}}-\phi(\alpha)\right), \\
\frac{1}{\sqrt{2 \pi}} \int_{0}^{\alpha} \exp \left(-\frac{1}{2} x^{2}-\frac{2}{3 \sqrt{s}} x\right) \mathrm{d} x=\mathrm{e}^{2 / 9 s}\left(\Phi\left(\frac{2}{3 \sqrt{s}}+\alpha\right)-\Phi\left(\frac{2}{3 \sqrt{s}}\right)\right) .
\end{gathered}
$$

Combining (44)-(48) yields the following result.

Theorem 7. For $\lambda<s$, the CDF of the Poisson distribution is bounded by

$$
\begin{aligned}
& \mathrm{P}\left(A_{\lambda} \leq s\right) \leq p(s)\left(\frac{1}{2}+\frac{2}{3 \sqrt{2 \pi s}}+\frac{1}{24 s}+\mathrm{e}^{2 / 9 s}\left(\Phi\left(\frac{2}{3 \sqrt{s}}+\alpha\right)-\Phi\left(\frac{2}{3 \sqrt{s}}\right)\right)\right), \\
& \mathrm{P}\left(A_{\lambda} \leq s\right) \geq p(s)\left(\Phi(\alpha)+\frac{2}{3 \sqrt{s}} \phi(\alpha)+\frac{1}{24 s}-\frac{4}{135 \sqrt{2 \pi} s^{3 / 2}}\right) .
\end{aligned}
$$

\section{The Erlang B formula}

We now utilize the results on $\mathrm{P}\left(A_{\lambda} \leq s\right)$ to derive similar results for the Erlang $\mathrm{B}$ formula. From (1) we see that the probability $\mathrm{P}\left(\bar{A}_{\lambda}=s\right)$ needs to be written in a different form. That is,

$$
\begin{aligned}
\mathrm{P}\left(A_{\lambda}=s\right) & =\mathrm{e}^{-\lambda} \frac{\lambda^{s}}{s !} \\
& =\mathrm{e}^{s(1-\rho+\ln \rho)} \frac{s^{s} \mathrm{e}^{-s}}{s !} \\
& =\mathrm{e}^{-\alpha^{2} / 2} \frac{1}{\sqrt{2 \pi s}} p(s) \\
& =\phi(\alpha) p(s) \frac{1}{\sqrt{s}} .
\end{aligned}
$$




\subsection{Expansions for Erlang's B}

Combining (1), (49), and the expansion for $\mathrm{P}\left(A_{\lambda} \leq s\right)$ yields the following result.

Theorem 8. For $s=\lambda+\beta \sqrt{\lambda}$ with $\beta$ some fixed real number, there exists, as $s$ tends to $\infty$, the representation

$$
\begin{aligned}
B(s, \lambda)^{-1} & \sim \frac{\sqrt{s}}{\phi(\alpha)} \sum_{n=0}^{\infty}(n+1) a_{n+1} \chi_{n}(\alpha)\left(\frac{1}{\sqrt{s}}\right)^{n} \\
& =\frac{\sqrt{s} \Phi(\alpha)}{\phi(\alpha)}+\frac{2}{3}+\frac{1}{12 \sqrt{s}}\left(\frac{\Phi(\alpha)}{\phi(\alpha)}-\alpha\right)+\cdots
\end{aligned}
$$

Jagerman [12] derived a related expansion.

Theorem 9. ([12, Theorem 14].) For $\lambda=s+\gamma \sqrt{s}$ with $\gamma$ some fixed real number, there exists, as $s$ tends to $\infty$, the representation

$$
B(s, \lambda)^{-1} \sim \sum_{n=0}^{\infty} v_{n}(\gamma)\left(\frac{1}{\sqrt{s}}\right)^{n-1},
$$

where

$$
\begin{aligned}
& v_{0}(\gamma)=\frac{\Phi(-\gamma)}{\phi(\gamma)} \\
& v_{1}(\gamma)=\frac{2}{3}+\frac{1}{3} \gamma^{2}-\frac{1}{3} \gamma^{3} v_{0}(\gamma), \\
& v_{2}(\gamma)=-\frac{1}{18} \gamma^{5}-\frac{7}{36} \gamma^{3}+\frac{1}{12} \gamma+\left(\frac{1}{18} \gamma^{6}+\frac{1}{4} \gamma^{4}+\frac{1}{12}\right) v_{0}(\gamma) .
\end{aligned}
$$

There are some marked differences between our expansion and that of Jagerman [12]. First, Jagerman sets the arrival rate $\lambda$ according to $\lambda=s+\gamma \sqrt{s}$ whereas we set the number of servers according to $s=\lambda+\beta \sqrt{\lambda}$. The constants $\beta$ and $\gamma$ are very much related, though, since

$$
\beta=\frac{s-\lambda}{\sqrt{\lambda}}, \quad \gamma=\frac{\lambda-s}{\sqrt{s}}=-\beta \rho^{1 / 2},
$$

so that $\gamma \downarrow-\beta$ as $\rho$ tends to 1 . Perhaps a more important difference is that we change our constant $\beta$ into $\alpha$. Since

$$
\frac{1}{2} \alpha^{2}=s \sum_{n=2}^{\infty} \frac{(1-\rho)^{n}}{n},
$$

we have, for large values of $s, \alpha \approx \sqrt{s}(1-\rho)=-\gamma \approx \beta$. A comparison between (50) and (51) is made in Table 2 for $s=\lambda+\beta \sqrt{\lambda}$ and $\beta=1$. We denote by (50)-1 and (50)-3 the approximations that follow from the first term and the first three terms of the asymptotic expansion in (50), respectively. The leading term (50)-1 is generally closer to $B(s, \lambda)$ than the leading term (51)-1. Both expansions benefit from taking larger values of $s$. When three terms are included, both expansions give excellent results, although for moderate values of $s$, expansion (50) seems slightly more accurate.

If $s=\lambda / c$ for some $c<1$ then $\alpha$ tends to $\infty$ as $\lambda$ tends to $\infty$. The approximation (50)-1 behaves as $\phi(\alpha) / \sqrt{s}$ as $s$ tends to $\infty$. It is easy to see that also $B(s, c s) \sim \phi(\alpha) / \sqrt{s}$ in this case. Consequently, (50)-1 is not only sharp in the QED regime, but also in the regime where the system load stays fixed, which is also known as the quality driven regime [4]. This is another reason why using $\alpha$ is preferable over $\beta$ or $-\gamma$. 
TABLE 2: Asymptotic expansions for $B(s, \lambda)$ with $s=\lambda+\beta \sqrt{\lambda}$ and $\beta=1$.

\begin{tabular}{rcccccccc}
\hline \multicolumn{1}{c}{$s$} & \multicolumn{1}{c}{$\lambda$} & $\alpha$ & $\gamma$ & $B(s, \lambda)$ & $(50)-1$ & $(51)-1$ & $(50)-3$ & $(51)-3$ \\
\hline 1 & 0.3820 & 0.8299 & -0.6180 & 0.2764 & 0.3548 & 0.4504 & 0.2739 & 0.2889 \\
2 & 1.0000 & 0.8790 & -0.7071 & 0.2000 & 0.2366 & 0.2890 & 0.1993 & 0.2057 \\
3 & 1.6972 & 0.9012 & -0.7522 & 0.1645 & 0.1880 & 0.2243 & 0.1642 & 0.1679 \\
5 & 3.2087 & 0.9236 & -0.8011 & 0.1282 & 0.1417 & 0.1642 & 0.1280 & 0.1298 \\
10 & 7.2984 & 0.9462 & -0.8543 & 0.0910 & 0.0974 & 0.1090 & 0.0909 & 0.0915 \\
20 & 16.0000 & 0.9622 & -0.8944 & 0.0644 & 0.0675 & 0.0734 & 0.0644 & 0.0646 \\
30 & 25.0000 & 0.9692 & -0.9129 & 0.0526 & 0.0546 & 0.0586 & 0.0526 & 0.0527 \\
50 & 43.4113 & 0.9762 & -0.9318 & 0.0407 & 0.0419 & 0.0443 & 0.0407 & 0.0408 \\
100 & 90.4875 & 0.9832 & -0.9512 & 0.0288 & 0.0294 & 0.0306 & 0.0288 & 0.0288 \\
200 & 186.3490 & 0.9881 & -0.9653 & 0.0204 & 0.0206 & 0.0213 & 0.0204 & 0.0204 \\
300 & 283.1723 & 0.9903 & -0.9715 & 0.0166 & 0.0168 & 0.0172 & 0.0166 & 0.0166 \\
500 & 478.1337 & 0.9925 & -0.9779 & 0.0129 & 0.0130 & 0.0132 & 0.0129 & 0.0129 \\
\hline
\end{tabular}

\subsection{Bounds for Erlang's B}

We have already given Theorem 1, in which the lower bound (5) follows immediately from (27) and (49). The upper bound (4) requires (26), (49), and the inequality $p(s) \geq 1-1 / 12 s$.

Theorem 4 gives the following result.

Theorem 10. For $\lambda>0$ and $s \in \mathbb{N}$, the reciprocal of Erlang's $B$ is bounded by

$$
\begin{aligned}
& B(s, \lambda)^{-1} \leq \frac{\Phi(\alpha+2 / 3 \sqrt{s}) \mathrm{e}^{2 / 9 s} \sqrt{s}}{\phi(\alpha)}, \\
& B(s, \lambda)^{-1} \geq \frac{\Phi(\alpha+2 / 3 \sqrt{s}) \mathrm{e}^{2 / 9 s} \sqrt{s}}{\phi(\alpha)}-\frac{\sqrt{s}\left(\mathrm{e}^{2 / 9 s}-1\right)}{\phi(\alpha)} .
\end{aligned}
$$

The next result follows immediately from Theorem 6 and sharpens Theorem 1 for $\lambda \geq s$.

Theorem 11. For $\lambda \geq s \in \mathbb{N}$, the reciprocal of Erlang's $B$ is bounded by

$$
\begin{aligned}
& B(s, \lambda)^{-1} \leq \frac{\Phi(\alpha) \sqrt{s}}{\phi(\alpha)}+\frac{2}{3}+\frac{\Phi(\alpha)-\alpha \phi(\alpha)}{12 \phi(\alpha) \sqrt{s}} \\
& B(s, \lambda)^{-1} \geq \frac{\Phi(\alpha) \sqrt{s}}{\phi(\alpha)}+\frac{2}{3}+\frac{\Phi(\alpha)-\alpha \phi(\alpha)}{12 \phi(\alpha) \sqrt{s}}-\frac{4+2 \alpha^{2}}{135 s}
\end{aligned}
$$

Likewise, for $\lambda<s$, a sharper version of Theorem 1 can be obtained from Theorem 7 .

In Table 3 we present some results for increasing values of $s=\lambda+\beta \sqrt{\lambda}$ with $\beta=1$. In this regime all bounds are sharp, even for smaller values of $s$. As expected, Erlang's approximation (2) requires $s$ to be large. The precision of the bounds is partly due to changing $\beta$ into $\alpha$. Moreover, the bounds (4) and (5) include the first-order correction term $\frac{2}{3}$, whereas the bounds (52) and (53) shift the mean of the Gaussian distribution with $2 / 3 \sqrt{s}$; both corrections seem beneficial, although the bounds (4) and (5) are sharper than (52) and (53).

In Table 4 we present some results for $B(s, \lambda)$ and $s=10$. The bounds (4) and (5) perform well, although in the case in which $\lambda \geq s$ the bounds (54) and (55) are much sharper. Hence, in this regime it seems beneficial to include the second-order correction term. 
TABLE 3: Bounds on $B(s, \lambda)$ with $s=\lambda+\beta \sqrt{\lambda}$ and $\beta=1$.

\begin{tabular}{rccccccc}
\hline \multicolumn{1}{c}{$s$} & $\lambda$ & $B(s, \lambda)$ & $(2)$ & $(4)$ & $(5)$ & $(52)$ & $(53)$ \\
\hline 1 & 0.3820 & 0.2764 & 0.4653 & 0.2627 & 0.2870 & 0.2427 & 0.3086 \\
2 & 1.0000 & 0.2000 & 0.2876 & 0.1953 & 0.2044 & 0.1882 & 0.2127 \\
3 & 1.6972 & 0.1645 & 0.2208 & 0.1620 & 0.1671 & 0.1582 & 0.1718 \\
5 & 3.2087 & 0.1282 & 0.1606 & 0.1270 & 0.1294 & 0.1253 & 0.1317 \\
10 & 7.2984 & 0.0910 & 0.1065 & 0.0906 & 0.0914 & 0.0900 & 0.0923 \\
20 & 16.0000 & 0.0644 & 0.0719 & 0.0643 & 0.0646 & 0.0641 & 0.0649 \\
30 & 25.0000 & 0.0526 & 0.0575 & 0.0525 & 0.0527 & 0.0524 & 0.0529 \\
50 & 43.4113 & 0.0407 & 0.0437 & 0.0407 & 0.0408 & 0.0407 & 0.0409 \\
100 & 90.4875 & 0.0288 & 0.0302 & 0.0288 & 0.0288 & 0.0288 & 0.0289 \\
200 & 186.3490 & 0.0204 & 0.0211 & 0.0204 & 0.0204 & 0.0204 & 0.0204 \\
300 & 283.1723 & 0.0166 & 0.0171 & 0.0166 & 0.0166 & 0.0166 & 0.0166 \\
500 & 478.1337 & 0.0129 & 0.0132 & 0.0129 & 0.0129 & 0.0129 & 0.0129 \\
\hline
\end{tabular}

TABLE 4: Bounds on $B(s, \lambda)$ for $s=10$.

\begin{tabular}{rcccccc}
\hline$\lambda$ & $B(s, \lambda)$ & $(2)$ & $(4)$ & $(5)$ & $(54)$ & $(55)$ \\
\hline 1 & 0.0000 & 0.0000 & 0.0000 & 0.0000 & - & - \\
2 & 0.0000 & 0.0000 & 0.0000 & 0.0000 & - & - \\
3 & 0.0008 & 0.0001 & 0.0008 & 0.0008 & - & - \\
4 & 0.0053 & 0.0022 & 0.0053 & 0.0053 & - & - \\
5 & 0.0184 & 0.0148 & 0.0184 & 0.0185 & - & - \\
6 & 0.0431 & 0.0452 & 0.0430 & 0.0434 & - & - \\
7 & 0.0787 & 0.0910 & 0.0784 & 0.0792 & - & - \\
8 & 0.1217 & 0.1445 & 0.1210 & 0.1223 & - & - \\
9 & 0.1680 & 0.1995 & 0.1669 & 0.1689 & - & - \\
10 & 0.2146 & 0.2523 & 0.2129 & 0.2160 & 0.2145 & 0.2146 \\
11 & 0.2596 & 0.3013 & 0.2570 & 0.2617 & 0.2594 & 0.2596 \\
12 & 0.3019 & 0.3459 & 0.2978 & 0.3051 & 0.3016 & 0.3019 \\
13 & 0.3412 & 0.3862 & 0.3344 & 0.3456 & 0.3407 & 0.3412 \\
14 & 0.3773 & 0.4225 & 0.3656 & 0.3833 & 0.3766 & 0.3773 \\
15 & 0.4103 & 0.4552 & 0.3901 & 0.4181 & 0.4094 & 0.4104 \\
16 & 0.4406 & 0.4847 & 0.4057 & 0.4503 & 0.4393 & 0.4406 \\
17 & 0.4682 & 0.5114 & 0.4089 & 0.4801 & 0.4666 & 0.4683 \\
18 & 0.4935 & 0.5356 & 0.3959 & 0.5077 & 0.4914 & 0.4936 \\
19 & 0.5167 & 0.5576 & 0.3629 & 0.5332 & 0.5141 & 0.5169 \\
20 & 0.5380 & 0.5778 & 0.3098 & 0.5570 & 0.5348 & 0.5383 \\
\hline
\end{tabular}

\section{A conjecture of Ramanujan}

We now indicate how our framework for obtaining bounds on $\mathrm{P}\left(A_{\lambda} \leq s\right)$ may deal with a conjecture of Ramanujan. In 1911 Ramanujan set the problem of showing that

$$
\xi(n)=\frac{n !}{n^{n}}\left(\frac{1}{2} \mathrm{e}^{n}-\sum_{k=0}^{n-1} \frac{n^{k}}{k !}\right), \quad n=1,2, \ldots,
$$


lies between $\frac{1}{2}$ and $\frac{1}{3}$. A solution was outlined by Ramanujan in 1912 and complete proofs were published by Szegö in 1928 and Watson in 1929 (see [8]). In his first letter to Hardy dated 16 January 1913 Ramanujan made the stronger assertion that

$$
\xi(n)=\frac{1}{3}+\frac{4}{135(n+\tau(n))}, \quad \text { where } \frac{8}{45} \leq \tau(n) \leq \frac{2}{21} .
$$

This was finally proved by Flajolet et al. [8] in 1995 using singularity analysis.

The connection with our framework is easily seen from

$$
\begin{aligned}
\xi(n) & =\frac{1}{2 \mathrm{P}\left(A_{n}=n\right)}-B(n, n)^{-1}+1 \\
& =\frac{\sqrt{2 \pi n}}{2}\left(\frac{1}{p(n)}-1\right)-B(n, n)^{-1}+1+\frac{\sqrt{2 \pi n}}{2} .
\end{aligned}
$$

Hence, in order to prove (56) we need to bound $1 / p(n)-1$ and $B(n, n)^{-1}$. The former causes no problems, because sufficiently sharp bounds can be obtained from truncating the Stirling series for $\ln (1 / p(n))$. For $B(n, n)^{-1}$, we have, from (44) and (45), the bounds

$$
\begin{aligned}
& B(n, n)^{-1} \leq \frac{\sqrt{2 \pi n}}{2}+\frac{2}{3}+\frac{\sqrt{2 \pi}}{24 \sqrt{n}}, \\
& B(n, n)^{-1} \geq \frac{\sqrt{2 \pi n}}{2}+\frac{2}{3}+\frac{\sqrt{2 \pi}}{24 \sqrt{n}}-\frac{4}{135 n} .
\end{aligned}
$$

As it turns out, these bounds are not sharp enough to prove (56). Our framework then prescribes the search for sharper bounds on $y^{\prime}$. The bounds eventually leading to a new proof (and actually sharpening) of (56) are

$$
\begin{array}{rrr}
y^{\prime}(x) \leq & 1-\frac{2}{3} x+\frac{1}{12} x^{2}+\frac{2}{135} x^{3}+\frac{1}{864} x^{4}-\frac{1}{2835} x^{5}, & x \leq 0, \\
y^{\prime}(x) \geq & 1-\frac{2}{3} x+\frac{1}{12} x^{2}+\frac{2}{135} x^{3}+\frac{1}{864} x^{4}-\frac{1}{2835} x^{5}-\frac{139}{777600} x^{6} \\
& -\frac{1}{25515} x^{7}-\frac{571}{261273600} x^{8}+\frac{281}{151559100} x^{9}, & x \leq 0 .
\end{array}
$$

The proof of these bounds relies on Lemma 3, which yields a sufficient condition in terms of inequalities for (finite-degree) polynomials. Since the proof is rather tedious, it is not included here.

\section{Concluding remarks and outlook}

We took the Erlang B formula as a vehicle for presenting bounds on the Poisson distribution. Our primary motivations to do so were the historical relevance of the Erlang B formula, and the recent interest in the Halfin-Whitt regime and square-root staffing. Obviously, the Erlang B formula is just one example to which the bounds for the Poisson distribution can be applied. One other example is the Erlang $\mathrm{C}$ formula, representing the steady-state delay probability in the $\mathrm{M} / \mathrm{M} / s$ queue. The bounds and series expansion for the Erlang B formula carry over to the Erlang $C$ formula since $C(s, \lambda)^{-1}=\rho+(1-\rho) B(s, \lambda)^{-1}$. In a companion paper [14] we applied this connection and the results of this paper to analyze the accuracy of server staffing algorithms in the Halfin-Whitt regime; cf. [4]. We are also currently applying our methodology to obtain sharp bounds for the normalization constant in loss networks; cf. Kelly [17]. 
Since we started our analysis from (6), all the results presented in this paper for the Poisson distribution also hold for the incomplete gamma function. In particular, our bounds complement the work of Temme [21], [22], [23] on asymptotic expansions and inversion of the incomplete gamma function.

Finally, let us mention that our function $y$, which is closely related to the Lambert $W$-function, has many applications in pure and applied mathematics; see [6]. In some of these applications bounds on $y$, which can be derived from Lemma 2, might be helpful.

\section{Appendix A. Numerical evaluation of the function $y$}

In this appendix we discuss several approaches for evaluating the function $y$ (and its derivative through $\left.y^{\prime}=x / y-x\right)$ defined as the solution to

$$
-y(x)-\ln (1-y(x))=\frac{1}{2} x^{2}
$$

for real values of $x$. This is needed in the evaluation of the quasi-Gaussian form for the CDF of the Poisson distribution in (9). There is the result

$$
y(x)=1+W\left(-\exp \left(-\left(1+\frac{1}{2} x^{2}\right)\right)\right), \quad x>0,
$$

where $W$ is Lambert's function, given for small $|X|$ as the solution $W=W(X)$ of $W \mathrm{e}^{W}=X$.

The power series representation (10) is valid for $|x|<2 \sqrt{\pi}$. For values of $x$ larger than $2 \sqrt{\pi}$, we can use the representation

$$
y(x)=1-\sum_{m=1}^{\infty} \frac{m^{m-1}}{m ! \mathrm{e}^{m}} \mathrm{e}^{-m x^{2} / 2}, \quad|\arg (x)| \leq \frac{\pi}{4},
$$

obtained in [13]. Next we discuss two methods that can be used to evaluate $y$ for $x \leq 0$.

\section{A.1. Newton iteration}

We have

$$
y(x)=-\frac{1}{2} x^{2}-\ln (1-y(x))=-\frac{1}{2} x^{2}+\mathcal{O}\left(\ln \frac{1}{2} x^{2}\right),
$$

and, by one more iteration,

$$
y(x)=-\frac{x^{2}}{2}-\ln \left(1+\frac{1}{2} x^{2}\right)+\mathcal{O}\left(\frac{\ln x^{2} / 2}{x^{2} / 2}\right) .
$$

The Newton iteration for (59) is given by

$$
y_{n+1}=1+\left(\frac{1}{y_{n}}-1\right)\left(\ln \left(1-y_{n}\right)+\frac{1}{2} x^{2}\right), \quad n=0,1, \ldots
$$

We use, for $x \leq 0$, the starting value

$$
y_{0}=-\frac{1}{2} x^{2}-\ln \left(1+\frac{1}{2} x^{2}\right) .
$$

For the case in which $x=-1$, we find $y_{4}=-1.357676674$ to be correct to 9 decimal places. For the case in which $x=-10$, we find $y_{2}=-54.007468976$ to be correct to 9 decimal places. 


\section{A.2. Double series expansion valid for $x<-\sqrt{\pi}$}

Jeffrey et al. [15] considered, for $v \in \mathbb{R}$ and large and positive $w$, the positive solution $z=\Psi_{v}(w)$ of the equation

$$
z^{v} \mathrm{e}^{z}=w
$$

In [15] it was shown that $\Psi_{v}(x)$ has an expansion

$$
\Psi_{v}(w)=L_{1}-v L_{2}+v \sum_{n=1}^{\infty} \frac{v^{n}}{L_{1}^{n}} \sum_{m=1}^{n}(-1)^{n+m}\left[\begin{array}{c}
n \\
n-m+1
\end{array}\right] \frac{L_{2}^{m}}{m !},
$$

where $L_{1}=\ln w, L_{2}=\ln \ln w$, and $\left[\begin{array}{c}n \\ m\end{array}\right]$ are Stirling cycle numbers, and the double series converges when $w>(|v| \mathrm{e})^{|v|}$ for the case in which $|\nu| \geq 1$. Now it holds, for $x<0$, that

$$
y(x)=1-\frac{1}{\mathrm{e}} \Psi_{-\mathrm{e}}\left(\mathrm{e}^{\mathrm{e} x^{2} / 2}\right)
$$

hence, the theory in [15] can be used to compute $y(x)$ for

$$
\exp \left(\frac{1}{2} \mathrm{e} x^{2}\right)>\exp (2 \mathrm{e})
$$

i.e. for $x<-2$. In fact, we have been able to sharpen the convergence results in [15]. In particular, for the case in which $\alpha=-\mathrm{e}$, which we have here, we can show that (60) converges exponentially if and only if $w>\exp \left(\frac{1}{2} \pi\right.$ e), i.e. if and only if $x<-\sqrt{\pi}$.

With

$$
u=\frac{1}{2} x^{2} \quad \text { and } \quad \tau=\frac{1+\ln u}{u}
$$

we obtain, from (61) and (60),

$$
y(x)=-u-\ln u-\sum_{n=0}^{\infty} \sum_{m=0}^{n} d_{n, n-m} u^{-m} \tau^{n-m} .
$$

Table 5 displays some of the coefficients and Figure 3 shows some numerical results of (62) for several truncation levels $n=N$.

TABLE 5: First few coefficients $d_{k, l}$.

\begin{tabular}{ccccccc}
\hline \multirow{8}{*}{$k$} & $d_{k, l}$ \\
\cline { 2 - 7 } & $l=0$ & $l=1$ & $l=2$ & $l=3$ & $l=4$ & $l=5$ \\
\hline 0 & 0 & 1 & $-\frac{1}{2}$ & $\frac{1}{3}$ & $-\frac{1}{4}$ & $\frac{1}{5}$ \\
1 & 0 & 1 & $-\frac{3}{2}$ & $\frac{11}{6}$ & $-\frac{25}{12}$ & $\frac{137}{60}$ \\
2 & 0 & 1 & -3 & $\frac{35}{6}$ & $-\frac{75}{8}$ & $\frac{203}{15}$ \\
3 & 0 & 1 & -5 & $\frac{85}{6}$ & $-\frac{245}{8}$ & $\frac{1241}{22}$ \\
4 & 0 & 1 & $-\frac{15}{2}$ & $\frac{175}{6}$ & $-\frac{245}{3}$ & $\frac{7483}{40}$ \\
5 & 0 & 1 & $-\frac{21}{2}$ & $\frac{161}{3}$ & -189 & $\frac{21091}{40}$ \\
\hline
\end{tabular}




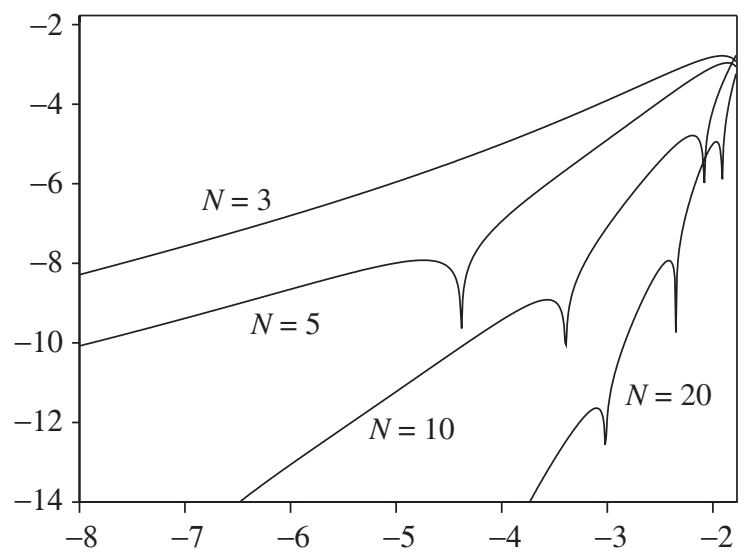

FIGURE 3: Relative error on a log-scale in estimating $y(x)$ for $x \in[-8,-\sqrt{\pi})$ through (62) truncated at $n=N$.

\section{Acknowledgements}

We are grateful to an anonymous referee for a careful examination of this paper. The research of Johan van Leeuwaarden was supported by an NWO VENI grant. The research of Bert Zwart was supported in part by NSF grants CMMI-0727400 and CNS-0718701.

\section{References}

[1] Atar, R., Mandelbaum, A. And Shaikhet, G. (2006). Queueing systems with many servers: null controllability in heavy traffic. Ann. Appl. Prob. 16, 1764-1804.

[2] Barndorff-Nielsen, O. E. And Cox, D. R. (1990). Asymptotic Techniques for Use in Statistics. Chapman and Hall, London.

[3] Bhattacharya, R. N. and Rao, R. R. (1976). Normal Approximations and Asymptotic Expansions. John Wiley, New York.

[4] Borst S., Mandelbaum, A. and Reiman, M. (2004). Dimensioning large call centers. Operat. Res. 52, $17-34$.

[5] Brockmeyer, E., Halstrøm, H. L. and Jensen, A. (1948). The life and works of A. K. Erlang. Trans. Danish Acad. Tech. Sci. 1948, 277pp.

[6] Corless, R. M. et al. (1996). On the Lambert W-function. Adv. Comput. Math. 5, 329-359.

[7] Feller, W. (1966). An Introduction to Probability Theory and Its Applications, Vol. II. John Wiley, New York.

[8] Flajolet, P., Grabner, P. J., Kirschenhofer, P. and Prodinger, H. (1995). On Ramanujan's Q-function. J. Comput. Appl. Math. 58, 103-116.

[9] Gans, N., Koole, G. and Mandelbaum, A. (2003). Telephone call centers: tutorial, review and research prospects. Manufacturing Service Operat. Manag. 5, 79-141.

[10] Halfin, S. and Whitt, W. (1981). Heavy-traffic limits for queues with many exponential servers. Operat. Res. 29, 567-588.

[11] Hwang, H. K. (1997). Asymptotic estimates of elementary probability distributions. Studies Appl. Math. 4, 339-417.

[12] Jagerman, D. (1974). Some properties of the Erlang loss function. Bell System Tech. J. 53, 525-551.

[13] Janssen, A. J. E. M., van LeeuwaArden, J. S. H. And Zwart, B. (2007). Corrected asymptotics for a multiserver queue in the Halfin-Whitt regime. Submitted.

[14] Janssen, A. J. E. M., van LeeuwaArden, J. S. H. And Zwart, B. (2007). Corrected server staffing by expanding Erlang C. Submitted.

[15] Jeffrey, D. J., Corless, R. M., Hare, D. E. G. and Knuth, D. E. (1995). Sur l'inversion de $y^{\alpha} \mathrm{e}^{y}$ au moyen des nombres de Stirling associés. C. R. Acad. Sci. Paris 320, 1449-1452.

[16] Johnson, N. L., Kotz, S. And Kemp, A. W. (1992). Univariate Discrete Distributions, 2nd edn. John Wiley, New York. 
[17] Kelly, F. P. (1991). Loss networks. Ann. Appl. Prob. 1, 319-378.

[18] Michel, R. (1993). On Berry-Esseen bounds for the compound Poisson distribution. Insurance Math. Econom. 13, 35-37.

[19] Petrov, V. V. (1995). Limit Theorems of Probability Theory. Clarendon Press, Oxford.

[20] ReEd, J. (2006). The G/GI/N queue in the Halfin-Whitt regime. Submitted.

[21] Temme, N. M. (1979). The asymptotic expansion of the incomplete gamma functions. SIAM J. Math. Anal. 10, 757-766.

[22] Temme, N. M. (1992). Asymptotic inversion of incomplete gamma functions. Math. Comput. 58, 755-764.

[23] Temme, N. M. (1996). Special Functions. John Wiley, New York. 